\title{
Landau Effective Interaction between Quasiparticles in a Bose-Einstein Condensate
}

\author{
A. Camacho-Guardian* and Georg M. Bruun \\ Department of Physics and Astronomy, Aarhus University, Ny Munkegade, DK-8000 Aarhus C, Denmark
}

(Received 19 December 2017; revised manuscript received 28 February 2018; published 15 August 2018)

Landau's description of the excitations in a macroscopic system in terms of quasiparticles stands out as one of the highlights in quantum physics. It provides an accurate description of otherwise prohibitively complex many-body systems and has led to the development of several key technologies. In this paper, we investigate theoretically the Landau effective interaction between quasiparticles, so-called Bose polarons, formed by impurity particles immersed in a Bose-Einstein condensate (BEC). In the limit of weak interactions between the impurities and the BEC, we derive rigorous results for the effective interaction. They show that it can be strong even for a weak impurity-boson interaction, if the transferred momentumenergy between the quasiparticles is resonant with a sound mode in the BEC. We then develop a diagrammatic scheme to calculate the effective interaction for arbitrary coupling strengths, which recovers the correct weak-coupling results. Using this scheme, we show that the Landau effective interaction, in general, is significantly stronger than that between quasiparticles in a Fermi gas, mainly because a BEC is more compressible than a Fermi gas. The interaction is particularly large near the unitarity limit of the impurity-boson scattering or when the quasiparticle momentum is close to the threshold for momentum relaxation in the BEC. Finally, we show how the Landau effective interaction leads to a sizable shift of the quasiparticle energy with an increasing impurity concentration, which should be detectable with presentday experimental techniques.

DOI: 10.1103/PhysRevX.8.031042

Subject Areas: Atomic and Molecular Physics

\section{INTRODUCTION}

Landau's quasiparticle theory represents a powerful framework for making accurate predictions about quantum many-body systems [1,2]. The quasiparticle concept dramatically reduces the complexity of the problem, and it is exploited across many areas of physics. The quasiparticle framework has led to technological breakthroughs such as semiconductor devices and superconductors, and it is central for the development of future quantum technologies. The effective interaction between quasiparticles plays a key role in Landau's theory, where it determines both thermodynamic and dynamical properties. Interactions between quasiparticles are observed in liquid-helium mixtures [3], and they are the origin of important effects such as conventional and high- $T_{c}$ superconductivity, where the interaction is mediated by lattice vibrations and spin fluctuations, respectively $[4,5]$. Likewise, the fundamental

\footnotetext{
* Corresponding author. acamacho@phys.au.dk

Published by the American Physical Society under the terms of the Creative Commons Attribution 4.0 International license. Further distribution of this work must maintain attribution to the author(s) and the published article's title, journal citation, and DOI.
}

interaction is caused by the exchange of gauge bosons in particle physics [6].

The experimental realization of highly populationimbalanced atomic gases, where the minority (impurity) atoms form quasiparticles, has significantly advanced our understanding of this fundamental topic. In particular, since the interaction between the impurity atoms and the majority atoms can be tuned using Feshbach resonances [7], one can study quasiparticle physics systematically and in regimes never realized before. Impurity atoms were first realized experimentally in degenerate Fermi gases, where they form quasiparticles called Fermi polarons [8-11]. We now have a good understanding of the Fermi polaron, even for strong interactions between the impurity and the Fermi gas [12-19]. Recently, two experiments observed long-lived quasiparticles formed by impurity atoms in a Bose-Einstein condensate $[20,21]$, following an earlier experiment on impurities in a one-dimensional (1D) Bose gas [22]. These quasiparticles, called Bose polarons, have been analyzed theoretically using a wide range of techniques [23-35].

Most of the theoretical and experimental studies in atomic gases focus on the properties of a single polaron, and much less is known about the interaction between polarons. In light of its key importance, a natural question is then whether the great flexibility of atomic-gas experiments can be used to obtain new insights into Landau's effective interaction, like it did concerning the properties of a single 
polaron. Studies of the Landau effective interaction have been limited to vanishing momenta using perturbation theory [36], and using variational and diagrammatic techniques for Fermi polarons [11,14,37], or to the case of two fixed impurities in real space using perturbation theory and variational techniques [38-40].

In this paper, we systematically investigate the Landau effective interaction between Bose polarons, both as a function of momenta and as a function of the bosonimpurity interaction strength. We derive rigorous results for a weak interaction using perturbation theory. It is shown that, even in this limit, the interaction between two polarons can be strong when the momentum-energy exchange is resonant with a sound mode in the BoseEinstein condensate (BEC). Our perturbative calculation is then generalized to arbitrary interaction strengths using a diagrammatic resummation scheme. We show that the Landau effective interaction, in general, is much stronger than that between Fermi polarons. The interaction is particularly strong when the boson-impurity interaction is close to the unitarity limit or when the momentum of one of the polarons approaches the onset of momentum relaxation caused by scattering bosons out of the BEC. The strong effective interaction is then demonstrated to give rise to a substantial shift in the polaron energy as a function of the impurity concentration. We conclude by discussing how such effects can be observed experimentally.

\section{BOSE POLARONS}

We consider mobile impurities of mass $m$, either bosonic or fermionic, immersed in a gas of bosons of mass $m_{B}$. The Hamiltonian of the system is

$$
\begin{aligned}
H= & \sum_{\mathbf{k}} \epsilon_{\mathbf{k}}^{B} b_{\mathbf{k}}^{\dagger} \hat{b}_{\mathbf{k}}+\frac{g_{B}}{2 V} \sum_{\mathbf{k}, \mathbf{k}^{\prime} \mathbf{q}} b_{\mathbf{k}+\mathbf{q}}^{\dagger} b_{\mathbf{k}^{\prime}-\mathbf{q}}^{\dagger} b_{\mathbf{k}^{\prime}} b_{\mathbf{k}} \\
& +\sum_{\mathbf{k}} \xi_{\mathbf{k}} c_{\mathbf{k}}^{\dagger} c_{\mathbf{k}}+\frac{g}{V} \sum_{\mathbf{k}, \mathbf{k}^{\prime} \mathbf{q}} b_{\mathbf{k}+\mathbf{q}}^{\dagger} c_{\mathbf{k}^{\prime}-\mathbf{q}}^{\dagger} c_{\mathbf{k}^{\prime}} b_{\mathbf{k}},
\end{aligned}
$$

where $b_{\mathbf{k}}^{\dagger}$ and $c_{\mathbf{k}}^{\dagger}$ create a boson and an impurity, respectively, with momentum $\mathbf{k}$, and $V$ is the volume. Here, $\epsilon_{\mathbf{k}}^{B}=$ $k^{2} / 2 m_{B}$ is the kinetic energy of the bosons, and $\xi_{\mathbf{k}}=$ $k^{2} / 2 m-\mu$ is the kinetic energy of the impurity atoms relative to their chemical potential $\mu$. The boson-boson and boson-fermion interactions are taken to be short range with strengths $g_{B}$ and $g$, and we assume that there is no direct interaction between the impurities. This assumption is due to the Pauli principle for fermionic impurities, whereas any direct interaction between bosonic impurities can easily be included at the mean-field level.

Below the critical temperature $T_{c}$, the bosons form a BEC with condensate density $n_{0}(T)$. The total density of the bosons is $n_{B}$, and the density $n$ of the impurities is taken to be so small that the bosons are unaffected. The BEC is accurately described by Bogoliubov theory, since we assume that the bosons are weakly interacting with a gas parameter $n_{B}^{1 / 3} a_{B} \ll 1$, where $a_{B}>0$ is the boson-boson scattering length. The normal and anomalous BEC Green's functions for the bosons are, respectively,

$$
\begin{aligned}
& G_{11}(\mathbf{k}, z)=\frac{u_{\mathbf{k}}^{2}}{z-E_{\mathbf{k}}}-\frac{v_{\mathbf{k}}^{2}}{z+E_{\mathbf{k}}}, \\
& G_{12}(\mathbf{k}, z)=\frac{u_{\mathbf{k}} v_{\mathbf{k}}}{z+E_{\mathbf{k}}}-\frac{u_{\mathbf{k}} v_{\mathbf{k}}}{z-E_{\mathbf{k}}},
\end{aligned}
$$

where $E_{\mathbf{k}}=\left[\epsilon_{\mathbf{k}}^{B}\left(\epsilon_{\mathbf{k}}^{B}+2 \mu_{B}\right)\right]^{1 / 2}$ is the Bogoliubov spectrum, $\mu_{B}=n_{0}(T) \mathcal{T}_{B}$ is the chemical potential of the bosons, and $u_{\mathbf{k}}^{2} / v_{\mathbf{k}}^{2}=\left[\left(\epsilon_{\mathbf{k}}^{B}+\mu_{B}\right) / E_{\mathbf{k}} \pm 1\right] / 2$ are the usual coherence factors. We define $\mathcal{T}_{B}=4 \pi a_{B} / m_{B}$.

The impurities interact with the BEC, forming quasiparticles denoted Bose polarons or simply polarons when there is no ambiguity. To avoid confusion, we remind the reader that Bose polarons refer to mobile impurities in a Bose gas. The impurities themselves can be either bosonic or fermionic as will indeed be discussed in detail below. A polaron with momentum $\mathbf{p}$ is described by the Green's function $G(\mathbf{p}, z)^{-1}=G_{0}(\mathbf{p}, z)^{-1}-\Sigma(\mathbf{p}, z), \quad$ where $\quad G_{0}(\mathbf{p}, z)^{-1}=$ $z-\xi_{\mathbf{p}}$ is the noninteracting Green's function and $\Sigma(\mathbf{p}, z)$ is the self-energy. The polaron energy $\varepsilon_{\mathbf{p}}$ is found by solving

$$
\varepsilon_{\mathbf{p}}=\xi_{\mathbf{p}}+\operatorname{Re} \Sigma\left(\mathbf{p}, \varepsilon_{\mathbf{p}}\right),
$$

where we assume that the imaginary part of the self-energy is small and the residue $Z_{\mathbf{p}}=\left.[1-\partial \Sigma(\mathbf{p}, z) / \partial z]^{-1}\right|_{z=\varepsilon_{\mathbf{p}}}$ of the quasiparticle is close to unity so that the polaron is well defined. In the following, we for simplicity focus on the case of an attractive interaction between the impurity and the BEC corresponding to a negative boson-impurity scattering length $a<0$. We calculate the self-energy $\Sigma(\mathbf{p}, z)$ using the finite-temperature field theory.

\section{LANDAU EFFECTIVE INTERACTION}

Even though there is no direct interaction between the impurities, two polarons interact via their effects on the surrounding $\mathrm{BEC}$ or, equivalently, due to the exchange of Bogoliubov sound modes. Landau's effective interaction between two polarons with momenta $\mathbf{p}_{1}$ and $\mathbf{p}_{2}$ is defined as [3]

$$
f\left(\mathbf{p}_{1}, \mathbf{p}_{2}\right)=Z_{\mathbf{p}_{1}} \frac{\delta \varepsilon_{\mathbf{p}_{1}}}{\delta n_{\mathbf{p}_{2}}}=Z_{\mathbf{p}_{1}} \frac{\delta \operatorname{Re} \Sigma\left(\mathbf{p}_{1}, \varepsilon_{\mathbf{p}_{1}}\right)}{\delta n_{\mathbf{p}_{2}}},
$$

where $n_{\mathbf{p}}$ is the quasiparticle distribution function and we use Eq. (3) in the second equality.

\section{A. Weak coupling}

We first consider the weakly interacting regime $k_{n}|a| \ll 1$, where $k_{n}=\left(6 \pi^{2} n_{B}\right)^{1 / 3}$ is the momentum scale set by the BEC. This regime allows us to derive analytical results valid to leading order in $k_{n} a$ and $n_{B}^{1 / 3} a_{B}$, which, in addition to providing important insights, also serve as a 
valuable guide for how to construct a strong coupling theory for the effective interaction.

To first order in the scattering length $a$, the polaron energy shift is $\mathcal{T}_{v} n_{B}$, which is independent of the impurity concentration. It therefore does not contribute to the effective interaction. Here $\mathcal{T}_{v}=2 \pi a / m_{r}$ is the boson-impurity zero-energy scattering matrix with $m_{r}=$ $m_{B} m /\left(m_{B}+m\right)$ the reduced mass.

The second-order contribution to the self-energy $\Sigma_{2}(\mathbf{p}, z)$ has been analyzed in detail for a single impurity at both zero and nonzero temperatures $[28,41]$. Here, we generalize this contribution to a finite impurity concentration $n$. In Fig. 1, we plot the second-order diagrams for the self-energy as an exchange (Fock) diagram with an induced interaction. This diagram illustrates that, while the induced interaction is inherently attractive in the static case, the sign of the corresponding Landau effective interaction obtained from Eq. (4) depends on the statistics of the impurity particles: It is attractive for bosonic impurities and repulsive for fermionic impurities in the case of a small energy transfer $[36,42]$. Note that the contribution to the self-energy that is independent of the impurity concentration, of course, does not depend on the statistics of the impurity particles, since it gives the energy shift of a single polaron [43]. To second order in $a$, it is enough to evaluate the self-energy at the unperturbed quasiparticle energy, i.e., setting $\varepsilon_{\mathbf{p}}=\xi_{\mathbf{k}}$ in Eq. (4) [28], and to take the residue to be unity. We obtain

$$
f\left(\mathbf{p}_{1}, \mathbf{p}_{2}\right)= \pm \mathcal{T}^{2} \chi\left(\mathbf{p}_{1}-\mathbf{p}_{2}, \xi_{\mathbf{p}_{1}}-\xi_{\mathbf{p}_{2}}\right),
$$

with $\chi(\mathbf{p}, z)=n_{0} p^{2} /\left[m_{B}\left(z^{2}-E_{p}^{2}\right)\right]$ the density-density correlation function of the $\mathrm{BEC}$ at a zero temperature. Here and in the following, the upper (lower) sign refers to bosonic (fermionic) impurities. This effective interaction is independent of the impurity concentration, and the mass ratio $m / m_{B}$ enters only through the factor $\mathcal{T}_{v}^{2} \propto m_{r}^{-2}$. Thus, the smaller the reduced mass, the stronger the effective interaction. Equation (5) recovers the well-known limit

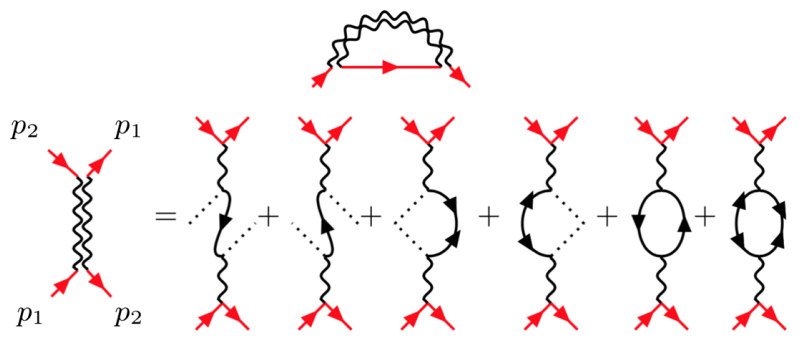

FIG. 1. Second-order self-energy for the Bose polaron as an exchange diagram, where the double wavy line is the secondorder induced interaction mediated by the BEC. Solid red lines correspond to the bare Green's function for the impurities, solid black lines are the normal and anomalous Bogoliubov Green's functions for the bosons, dashed lines correspond to condensate particles, and wavy lines represent the zero-energy bosonimpurity vacuum scattering matrix $\mathcal{T}_{v}$.

$$
\lim _{\mathbf{p}_{1}, \mathbf{p}_{2} \rightarrow 0} f\left(\mathbf{p}_{1}, \mathbf{p}_{2}\right)=\mp \frac{\mathcal{T}_{v}^{2}}{\mathcal{T}_{B}}=\mp\left(\frac{\partial \mu}{\partial n_{B}}\right)^{2} n_{B}^{2} \kappa
$$

where $\kappa=n_{B}^{-2} \partial n_{B} / \partial \mu_{B}$ is the compressibility of the BEC at a zero temperature. In the second equality, we use $\mu=$ $\mathcal{T}_{B} n_{B}$ to the lowest order so that the Landau interaction for vanishing momenta can be written in terms of thermodynamic derivatives $[14,36,42,44]$.

In Eq. (5), we do not include the last two "bubble" diagrams in Fig. 1. These diagrams are suppressed by a factor of $\left(n_{0} a_{B}^{3}\right)^{1 / 2}$ for $T \ll T_{c}[28,41]$ and, therefore, give a small contribution to the effective interaction except for very low momenta, where they give an unphysical divergence for $\lim _{\mathbf{p}_{1}, \mathbf{p}_{2} \rightarrow 0} f\left(\mathbf{p}_{1}, \mathbf{p}_{2}\right)$ for $T>0$. This divergence comes from an infrared pole in the distribution function of the thermally excited bosons $n_{\mathbf{k}}^{B}=1 /\left[\exp \left(E_{\mathbf{k}} / T\right)-1\right]$ as $k \rightarrow 0$. The interaction with thermally excited bosons is related to the complicated and largely unresolved problem of a systematic theory for a BEC at a finite temperature $[45,46]$, which is beyond the scope of the present paper. As we consider low temperatures here, we neglect these bubble diagrams.

From a general point of view, we expect the induced interaction to decrease with an increasing temperature. The reason is that the Bose gas becomes less compressible as particles are excited out of the BEC [47]. This effect will, however, be small for temperatures much smaller than the critical temperature of the BEC, as considered in this paper.

We plot in Fig. 2 the zero center-of-mass momentum (COM) Landau effective interaction $f(\mathbf{p},-\mathbf{p})$ given by Eq. (5), for a zero temperature, vanishing impurity concentration, unit mass ratio $m / m_{B}=1$, and $k_{n} a_{B}=0.2$. There are no retardation effects for a zero COM, and Eq. (5) gives the well-known Yukawa interaction. In real space, it is given by

$$
f(\mathbf{r})=-\frac{\mathcal{T}_{v}^{2} n_{0} m_{B}}{\pi} \frac{e^{-\sqrt{2} r / \xi}}{r},
$$

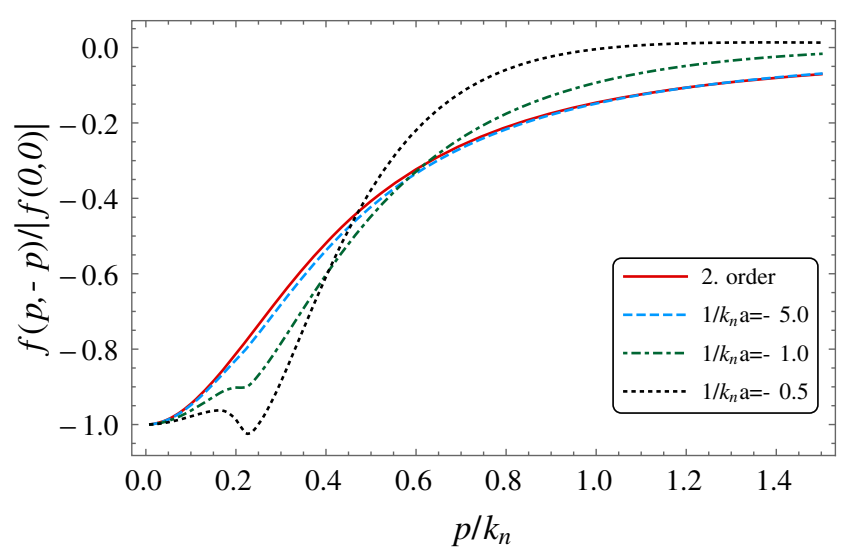

FIG. 2. The Landau effective interaction $f(\mathbf{p},-\mathbf{p})$ for a zero COM, vanishing impurity concentration, zero temperature, unit mass ratio $m / m_{B}=1$, and $k_{n} a_{B}=0.2$. We show the perturbative result (solid red curve), $1 / k_{n} a=-5.0$ (dashed blue curve), $1 / k_{n} a=-1.0$ (dot-dashed green curve), and $1 / k_{n} a=-0.5$ (short-dashed black curve). 
where $r$ is the distance between the impurities and $\xi=$ $\left(8 \pi a_{B} n_{B}\right)^{-1 / 2}$ is the BEC coherence length. In Fig. 2 and Eq. (7), we take the impurity to be bosonic, since this is natural for a unit mass ratio $m / m_{B}=1$. The fermionic case can be obtained by a simple sign change. Interestingly, we note that the weak-coupling result (7) is not obtained in a variational calculation of the interaction between two fixed impurities [40].

We plot in Figs. 3-5 the Landau effective interaction $f(0, \mathbf{p})$ calculated from Eq. (5), which gives the interaction between a zero-momentum polaron and a polaron with momentum $\mathbf{p}$ in the weak-coupling limit. As above, we take $k_{n} a_{B}=0.2, T=0$, and a vanishing impurity concentration $n$. Figures 3-5 correspond to the mass ratios $m / m_{B}=1$, $m / m_{B}=40 / 7$, and $m / m_{B}=40 / 87$, which describe the situation for the experiments by Aarhus [20], Innsbruck [48], and JILA [21], respectively. For unit mass ratio $m / m_{B}=1$, we have from Eq. (5) $f(0, \mathbf{p})=-\mathcal{T}_{v}^{2} / \mathcal{T}_{B}$ (bosonic impurity), which is simply a constant independent of momentum. For $m / m_{B}=40 / 87$ and $m / m_{B}=40 / 7$, on the other hand, there is a strong momentum dependence of $f(0, \mathbf{p})$. The interaction is repulsive for small momenta in both cases since we take the impurities to be fermionic to model the experiments in Refs. $[21,48]$. In the case of heavy impurities with $m / m_{B}=40 / 7$ shown in Fig. 4, the repulsive interaction decreases monotonically with the momentum. The Landau effective interaction $f(0, \mathbf{p})$ exhibits an interesting behavior in the case of light impurities with $m / m_{B}=40 / 87$, as shown in Fig. 5. It increases strongly with momentum $\mathbf{p}$ until $p^{2} / 2 m=E_{p}$. Here, it diverges even though the bosonimpurity interaction is weak. The reason is that the transferred momentum-energy $\left(p, p^{2} / 2 m\right)$ is resonant with a Bogoliubov sound mode in the BEC so that the densitydensity response of the BEC diverges. For a larger momentum where $p^{2} / 2 m>E_{p}$, the energy exchange between the polarons is above the Bogoliubov spectrum, which means that the density-density response function of the BEC

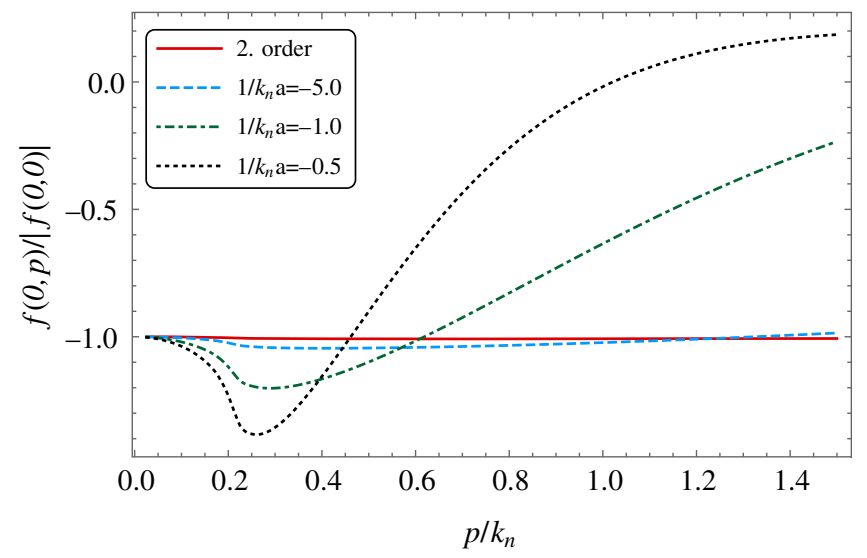

FIG. 3. The Landau effective interaction $f(0, \mathbf{p})$ for unit mass ratio $m / m_{B}=1$, vanishing impurity concentration, zero temperature, $k_{n} a_{B}=0.2$, and various impurity-boson scattering lengths. The second-order perturbative results are also plotted.

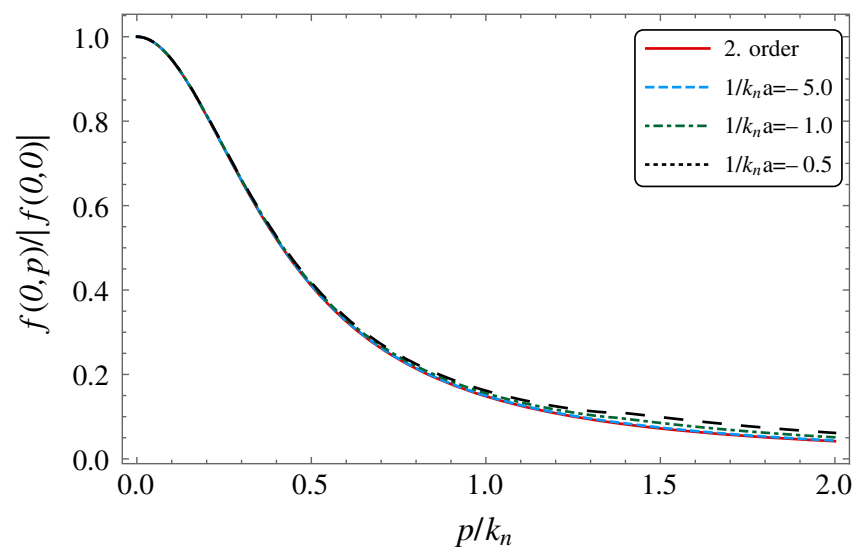

FIG. 4. The Landau effective interaction $f(0, \mathbf{p})$ for $m / m_{B}=$ $40 / 7$, vanishing impurity concentration, $T=0, k_{n} a_{B}=0.2$, and various impurity-boson scattering lengths. The second-order perturbative results are also plotted.

changes sign. The effective interaction between the polarons is consequently attractive even though the impurities are fermionic.

Note that this resonance effect happens only for light impurities with $m<m_{B}$, where it is possible to have $p^{2} / 2 m=E_{p}$. In reality, the divergence of the effective interaction when $p^{2} / 2 m=E_{p}$ is softened to a resonance structure for a finite impurity concentration, since the sound modes of the BEC are damped due to the scattering on the impurities. These scattering processes are not included in Bogoliubov theory. For the small impurity concentrations considered in this paper, the damping effects will, however, be negligible, and the broadening of the pole will be correspondingly narrow.

\section{B. Strong coupling}

A very powerful feature of atomic gases is that one can tune the interaction strength $k_{n} a$ over orders of magnitude using Feshbach resonances, and we therefore now consider

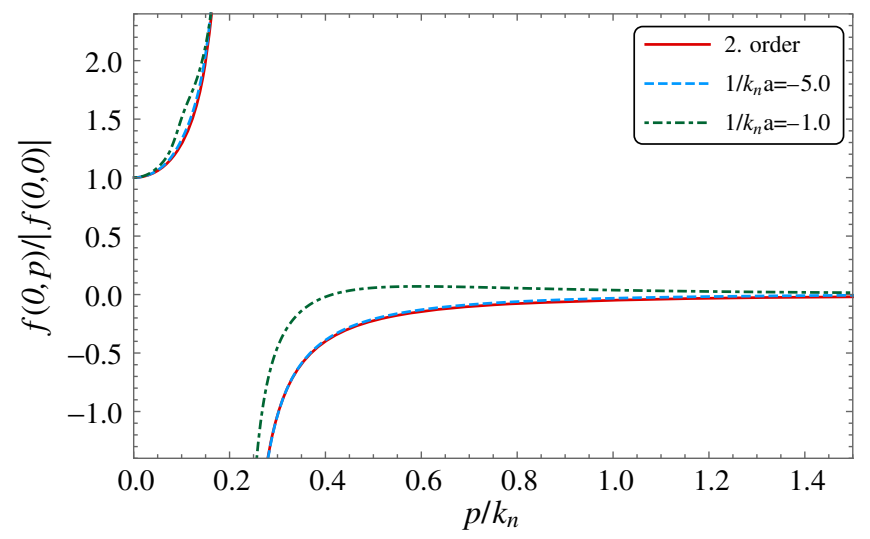

FIG. 5. The Landau effective interaction $f(0, \mathbf{p})$ for $m / m_{B}=$ 40/87, vanishing impurity concentration, $T=0, k_{n} a_{B}=0.2$, and various impurity-boson scattering lengths. The second-order perturbative results are also plotted. 
(a)

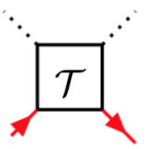

(b)

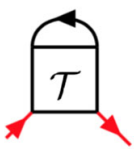

(b)

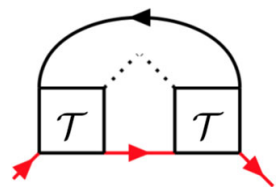

(c)

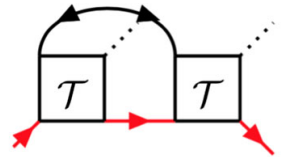

(d)

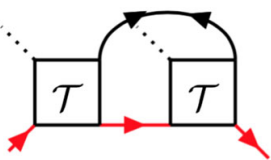

(e)

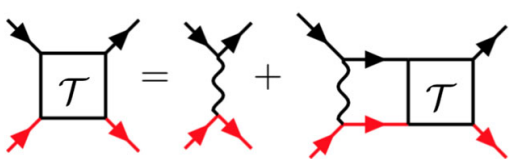

FIG. 6. (Top panel) Diagrams for the self-energy for a general boson-impurity interaction strength. (Bottom panel) Ladder approximation for the boson-impurity scattering matrix $\mathcal{T}(p)$.

the Landau effective interaction for an arbitrary impurityboson interaction strength. Here, one has to resort to approximations when calculating the self-energy, since there is no small parameter. The results obtained above for weak coupling serve as an important guide to construct a consistent theory.

In Fig. 6, we show the diagrams included in our selfenergy calculation, and Fig. 7 shows the corresponding diagrams they generate for the Landau effective interaction. These diagrams are obtained by taking the derivative $\delta \Sigma / \delta n_{\mathrm{p}}$ according to Eq. (4), which corresponds to removing an impurity line in the self-energy diagrams. Diagrams (a) and (b) in Fig. 6 represent the usual ladder approximation, which has been extensively used to describe atomic gases. In particular, it has recently been applied to describe the properties of a single Bose polaron [27]. The ladder approximation is, however, not sufficient to describe the Landau interaction between Bose polarons. This issue can be seen already in the weak-coupling limit, where the ladder approximation recovers only the first diagram in Fig. 1, which gives a qualitatively wrong result for the Landau effective interaction. Analogously, the ladder diagram in Fig. 6(a) generates only the first diagram in Fig. 7

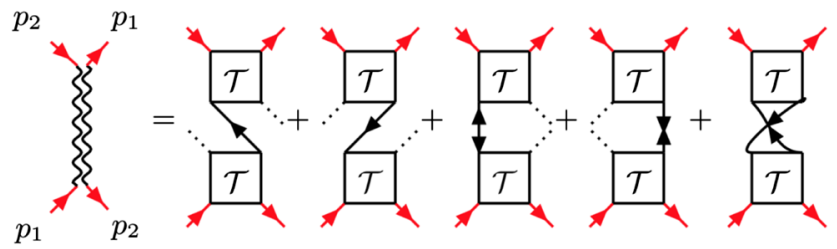

FIG. 7. Diagrams for the induced interaction obtained from the self-energy diagrams in Fig. 6. We omit the last diagram in the calculations. for strong coupling. While this term does recover the microscopic impurity-boson scattering in the ladder approximation, it fails to properly describe the propagation of density oscillations in the BEC. We therefore include diagrams (c)-(e) in Fig. 6 for the self-energy, which generate the last four diagrams in Fig. 7. These diagrams ensure that we describe the density oscillations in the BEC correctly and that we recover the correct weak-coupling result.

Similar diagrammatic schemes going beyond the ladder approximation were recently developed to analyze BoseFermi mixtures [49] and finite-temperature effects for a single polaron [50]. The diagrammatic scheme presented here is, however, the first to include diagrams (d) and (e) containing anomalous propagators, which is crucial for obtaining the correct induced interaction in the perturbative limit as well as for avoiding unphysical divergencies for strong interactions. The inadequacy of the ladder approximation for obtaining the correct induced interaction should be contrasted to the case of Fermi polarons, where it is sufficient to recover the correct weak-coupling result [14].

Two remarks are in order here. First, we do not include diagram (b) in Fig. 6, which generates the last diagram in Fig. 7 for the interaction. The reason is the same as for omitting the bubble diagrams in Fig. 1 for weak coupling. We exclude this diagram because it gives a small contribution to the self-energy and to the effective interaction for $T \ll T_{c}$ except for vanishing momenta, where it results in an unphysical divergence for the effective interaction. Second, the operation $\delta \Sigma / \delta n_{\mathbf{p}}$ generates additional contributions to the effective interaction, which are shown in Fig. 11 in the Appendix. These terms come from the dependence of the boson-impurity scattering matrix $\mathcal{T}$, given below in (9), on the impurity concentration $n$. In general, their contribution to the effective interaction is small, and we do not include them in the following.

The expression for the self-energy corresponding to diagrams (a) and (c)-(e) in Fig. 6 is

$$
\begin{aligned}
\Sigma(p)= & n_{0} \mathcal{T}(p)-n_{0} \sum_{k} G_{11}(k) \mathcal{T}^{2}(k+p) G(k+p) \\
& -2 n_{0} \mathcal{T}(p) \sum_{k} G_{12}(k) \mathcal{T}(k+p) G(k+p),
\end{aligned}
$$

where we define the four-momentum $p=(\mathbf{p}, z)$ and $\sum_{k}$ is shorthand for $T \sum_{i \omega_{n}} \int d^{3} k /(2 \pi)^{3}$ with $\omega_{n}=2 n T$ as the Bose Matsubara frequency. The boson-impurity scattering matrix is

$$
\mathcal{T}(p)=\frac{\mathcal{T}_{\nu}}{1-\mathcal{T}_{\nu} \Pi_{11}(p)},
$$

with $\Pi_{11}(p)=-\sum_{k} G_{11}(k) G(k+p)$ the in-medium pair propagator. In the Appendix, we provide a detailed 
expression for the self-energy after the Matsubara sum has been performed.

The induced interaction corresponding to the first four diagrams in Fig. 7 is

$$
\begin{aligned}
f\left(\mathbf{p}_{1}, \mathbf{p}_{2}\right)= & \pm n_{0} Z_{\mathbf{p}_{1}} Z_{\mathbf{p}_{2}}\left[\mathcal{T}^{2}\left(p_{1}\right) G_{11}\left(p_{1}-p_{2}\right)\right. \\
& +\mathcal{T}^{2}\left(p_{2}\right) G_{11}\left(p_{2}-p_{1}\right) \\
& \left.+2 \mathcal{T}\left(p_{1}\right) \mathcal{T}\left(p_{2}\right) G_{12}\left(p_{2}-p_{1}\right)\right]
\end{aligned}
$$

where $p_{i}=\left(\mathbf{p}_{i}, \varepsilon_{\mathbf{p}_{i}}\right)$ is the on-shell polaron momentumenergy. In deriving Eq. (10), we use the fact that the diagrams for $f\left(\mathbf{p}_{1}, \mathbf{p}_{2}\right)$ have to be evaluated with the external outgoing momenta $\mathbf{p}_{1}$ and $\mathbf{p}_{2}$ swapped compared to the ingoing momenta as indicated in Fig. 7, since the Landau interaction is caused by exchange processes. The factor of $Z_{\mathbf{p}_{2}}$ in Eq. (10) comes from the residue of the quasiparticle poles of the Green's functions inside the diagrams.

In Fig. 2, we plot $f(\mathbf{p},-\mathbf{p})$ for $1 / k_{n} a=-5,1 / k_{n} a=-1$, and $1 / k_{n} a=-0.5$, and all other parameters are as in the perturbative limit discussed in Sec. III A. We assume that the quasiparticle residues are independent of the momentum, i.e., $Z_{\mathbf{p}} \simeq Z_{0}$, so that they cancel out when normalizing by $f(0,0)$. The Landau interaction is close to the secondorder Yukawa result for $1 / k_{n} a=-5$. This result confirms that our diagrammatic resummation scheme illustrated in Figs. 6 and 7 recovers the correct weak-coupling limit. On the other hand, we see from Fig. 2 that the Landau interaction is quite different from the Yukawa form for the stronger coupling strengths $1 / k_{n} a=-1$ and $1 / k_{n} a=$ -0.5 . In particular, it develops a minimum around $p \simeq m c$ corresponding to the maximum attraction, where $c=$ $\left(4 \pi n_{0} a_{B}\right)^{1 / 2} / m_{B}$ is the speed of sound in the BEC. This momentum gives the threshold for momentum relaxation at a zero temperature: For momenta $p>m c$, the polaron momentum can decrease by scattering bosons out of the condensate. These processes give rise to an imaginary part of the pair propagator $\Pi_{11}(p)$ in the scattering matrix Eq. (9) for $p>m c$, whereas $\Pi_{11}(p)$ is purely real for $p<m c$. At the threshold $p=m c$, the absolute value of $\Pi_{11}(p)$ is minimum. This value leads to a large bosonimpurity scattering amplitude and, therefore, a maximum magnitude of the effective interaction.

We remark that the physical origin of this maximum is distinct from the origin of the pole discussed in Sec. III A in connection with Fig. 5. It is purely a strong-coupling effect coming from the momentum dependence of the impurityboson scattering matrix, whereas the pole structure is present even for weak coupling where the scattering matrix is a constant, since it is caused by the transferred energymomentum being resonant with a Bogoliubov mode.

We see from Fig. 2 that the interaction $f(\mathbf{p},-\mathbf{p})$ decreases again for $p>m c$ and it even changes sign for large momenta for $1 / k_{n} a=-0.5$. This change is caused by a large imaginary part of the pair propagator $\Pi_{11}(p)$ in the scattering matrix. Physically, it reflects fast momentum relaxation of a polaron with a large momentum, which, however, also means that its lifetime is short, making the validity of Landau's quasiparticle description questionable.

We plot in Fig. 3 the effective interaction $f(0, \mathbf{p})$ for $1 / k_{n} a=-5.0,1 / k_{n} a=-1$, and $1 / k_{n} a=-0.5$, and all other parameters are as in the perturbative limit. Again, our diagrammatic scheme is close to the perturbative result $f(0, \mathbf{p})=-\mathcal{T}_{v}^{2} / \mathcal{T}_{B}$ for the weak-coupling strength $1 / k_{n} a=-5$. The slight momentum dependence for $1 / k_{n} a=-5.0$ reflects the weak momentum dependence of the scattering matrix Eq. (9), which is neglected in the perturbative calculation. Again, the Landau effective interaction is very different from the perturbative result for the stronger coupling strengths $1 / k_{n} a=-1$ and $1 / k_{n} a=-0.5$. We see that it depends strongly on the momentum and that there is a minimum, i.e., maximum attraction, for $p \simeq m c$. The reason is the same as for $f(\mathbf{p},-\mathbf{p})$ discussed above: The scattering is resonant when the polaron momentum is at the threshold for momentum relaxation. For $p \gtrsim m c$, the strength of the interaction decreases again with the momentum and even changes sign for strong coupling. Again, this change is, however, a sign that high-momentum polarons are strongly damped due to momentum relaxation so that they are not welldefined quasiparticles.

Figures 4 and 5 show $f(0, \mathbf{p})$ for the mass ratios $m / m_{B}=40 / 7$ and $m / m_{B}=40 / 87$ corresponding to fermionic impurities with all other parameters the same as in the perturbative limit. We see that the effects of strong interactions are less dramatic for these mass ratios. Strong correlations change the quantitative value of the effective Landau interaction, but the qualitative behavior is the same as in the perturbative limit. In particular, the effective interaction still diverges for light impurities when the transferred momentum-energy is resonant with a Bogoliubov sound mode at $p^{2} / 2 m=E_{p}$.

For all mass ratios, $f(0, \mathbf{p})$ and $f(\mathbf{p},-\mathbf{p})$ tend to zero for $|\mathbf{p}| \gg 1 / a$, since the scattering becomes suppressed for high momenta.

To illustrate how the strength of the Landau interaction depends on the boson-impurity coupling strength, we plot in Fig. $8 f(0,0) / Z_{0}^{2}$ as a function of $1 / k_{n} a$ for $T=0$, $n \rightarrow 0, m / m_{B}=40 / 7$, and three different boson-boson interaction strengths: $k_{n} a_{B}=0.1, \quad k_{n} a_{B}=0.15$, and $k_{n} a_{B}=0.2$. From Eq. (10), it follows that

$$
\frac{f(0,0)}{Z_{0}^{2}}= \pm \mathcal{T}^{2}\left(0, \varepsilon_{\mathbf{p}=0}\right) \chi(0,0)=\mp \frac{\mathcal{T}^{2}\left(0, \varepsilon_{\mathbf{p}=0}\right)}{\mathcal{T}_{B}},
$$

where the expression $\chi(0,0)$ should be understood as the limit $\lim _{\mathbf{p} \rightarrow 0 \chi}(\mathbf{p}, 0)$ of the density-density correlation function. Equation (6) clearly reduces to Eq. (6) in the weak-coupling perturbative regime. From Fig. 8, we see 


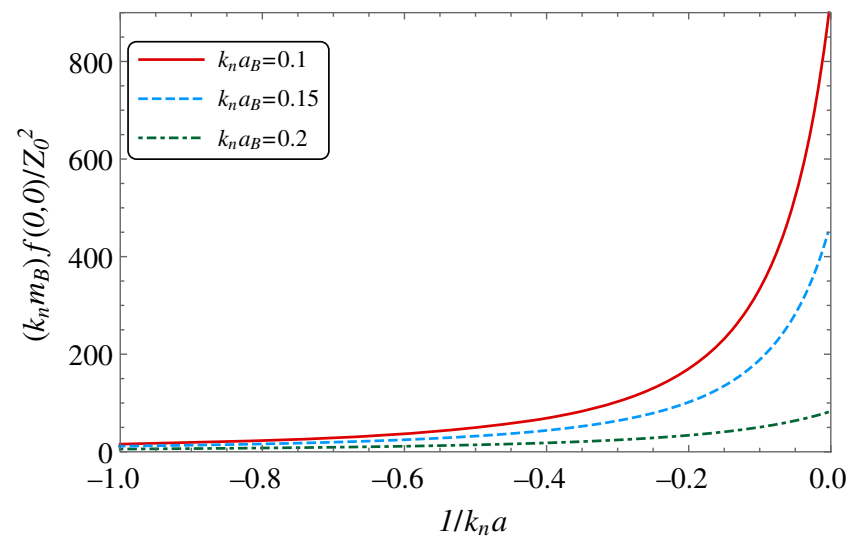

FIG. 8. The Landau effective interaction $f(0,0)$ as a function of $1 / k_{n} a$ for a vanishing impurity concentration, zero temperature, mass ratio $m / m_{B}=40 / 7$, and various boson-boson interaction strengths.

that $f(0,0)$ increases monotonically with $k_{n}|a|$ as expected. For weak coupling, we have $f(0,0) \propto a^{2}$, whereas it saturates to a maximum value for $k_{n}|a| \rightarrow \infty$, which depends on both the mass ratio and $a_{B}$. This value is much larger than the corresponding effective interaction between Fermi polarons with vanishing momenta in the unitarity limit. Indeed, Monte Carlo, variational, and ladder calculations yield $5.0 \lesssim m k_{F} f(0,0) \lesssim 7.1$ for Fermi polarons in the unitarity limit $[11,14,37]$, which is much smaller than what is shown in Fig. 8. We note that $f(0,0)$ remains large even when the factor $Z_{0}^{2}$ is included, since the quasiparticle residue for the Bose polaron residue remains significant for all scattering lengths shown here $[24,27,50]$.

The reasons for the larger value of $f(0,0)$ for the Bose polaron as compared to the Fermi polaron are twofold. First, a weakly interacting BEC is much more compressible than the Fermi gas so that it mediates density fluctuations more efficiently. This efficiency is reflected in the explicit $1 / a_{B}$ dependence in Eq. (11) coming from the fact that the more weakly interacting the $\mathrm{BEC}$, the more compressible it is. We also see this efficiency in Fig. 8, which shows that the effective interaction increases with decreasing $k_{n} a_{B}$. Second, both the impurity and the bosons have vanishing momenta in the scattering processes, giving rise to the effective interaction $f(0,0)$, since the bosons are scattered out of the condensate. This change gives rise to the $\mathcal{T}^{2}\left(0, \varepsilon_{\mathbf{p}=0}\right)$ dependence in Eq. (11), which significantly increases the effective interaction, since the scattering amplitude, in general, is larger for lower momenta. In contrast, the impurity-fermion scattering leading to the Landau interaction between Fermi polarons is averaged over all momenta inside the Fermi sea.

Note, however, that $f(0,0)$ is not easy to detect directly. As we analyze in the next section, observables such as the total energy or the polaron energy involve momentum averages of $f\left(\mathbf{p}_{1}, \mathbf{p}_{2}\right)$, which mask a large value of $f(0,0)$. Also, a large value of the effective interaction means that higher-order processes not included in this calculation, such as the repeated exchange of phonons in a ladder series, are important. Such higher order might suppress the magnitude of $f(0,0)$, but it will likely remain large.

\section{DENSITY DEPENDENCE OF SELF-ENERGY}

The Landau effective interaction $f\left(\mathbf{p}_{1}, \mathbf{p}_{2}\right)$ is difficult to measure directly. For conventional Fermi liquids, momentum averages of $f\left(\mathbf{p}_{1}, \mathbf{p}_{2}\right)$ over the Fermi surface can be extracted from thermodynamic quantities as well as from the collective mode spectrum [3]. For atomic gases, it has been proposed to use a mixed dimensional Bose-Fermi system to detect the induced interaction mediated by a BEC [51]. We now discuss how Landau's effective interaction can be probed by measuring the dependence of the polaron energy on the impurity concentration.

It follows from Eq. (4) that a small change $\delta n_{\mathbf{k}}$ in the polaron distribution function gives rise to the change

$$
\delta \varepsilon_{\mathbf{p}}=\int \frac{d^{3} k}{(2 \pi)^{3}} f(\mathbf{p}, \mathbf{k}) \delta n_{\mathbf{k}}
$$

in the polaron energy. Thus, the dependence of $\varepsilon_{\mathbf{p}}$ on the impurity density $n$ is a direct consequence of the effective interaction. We write the change of a zero-momentum polaron energy from its value at zero impurity density as

$$
\Delta \varepsilon(x)=\varepsilon(x)-\varepsilon(0) .
$$

Here $x=n / n_{B}$ is the impurity concentration, and $\varepsilon(x)$ denotes the polaron energy at concentration $x$. We suppress the momentum label in Eq. (13) and in the following, since we consider a zero-momentum polaron from now on.

In the perturbative regime, the induced interaction given by Eq. (5) does not depend on the impurity concentration. The change in the $\mathbf{p}=0$ polaron energy due to a nonzero impurity concentration is then simply

$$
\Delta \varepsilon(x)= \pm \mathcal{T}_{v}^{2} \int \frac{d^{3} k}{(2 \pi)^{3}} n_{\mathbf{k}} \chi\left(\mathbf{k}, \xi_{\mathbf{k}}+\mu\right) .
$$

Equation (14) can be derived either directly from the second-order self-energy or from Eqs. (5) and (12). For a stronger impurity-boson interaction, the Landau effective interaction does depend on the impurity concentration, and Eq. (14) no longer holds.

For equal masses with $m=m_{B}$, Eq. (14) can easily be evaluated, yielding $\Delta \varepsilon(x)=\mp n \mathcal{T}_{v}^{2} / \mathcal{T}_{B}$. This result is a simple consequence of $f(0, \mathbf{p})=\mp \mathcal{T}_{v}^{2} / \mathcal{T}_{B}$ being constant in the perturbative limit for a unit mass ratio. The perturbative energy shift Eq. (14) can also be calculated analytically for any mass ratio $m / m_{B}$ for fermionic impurities at $T=0$. Using $n_{\mathbf{k}}=\Theta\left(k_{F}-k\right)$, where $k_{F} / k_{n}=x^{1 / 3}$ is the impurity Fermi momentum, we obtain 


$$
\begin{aligned}
\frac{\Delta \varepsilon(x)}{E_{n}}= & \left(k_{n} a\right)^{2} \frac{8}{3 \pi^{2}} \frac{(1+\alpha)^{2}}{\left(\alpha^{2}-1\right)^{3 / 2}}\left[\frac{k_{F}}{k_{n}} \sqrt{\alpha^{2}-1}\right. \\
& -\sqrt{\frac{2 \alpha^{2} m_{B} c^{2}}{E_{n}}} \arctan \left(\frac{k_{F}}{k_{n}} \sqrt{\left.\frac{E_{n}}{2 m_{B} c^{2}} \frac{\alpha^{2}-1}{\alpha^{2}}\right)}\right] \\
\simeq & \left(k_{n} a\right)^{2} \frac{1}{3 \pi} \frac{(1+\alpha)^{2}}{\alpha^{2}} \frac{x}{k_{n} a_{B}}=n \frac{\mathcal{T}_{v}^{2}}{\mathcal{T}_{B}} \frac{1}{E_{n}},
\end{aligned}
$$

where $\alpha=m / m_{B}$ is the mass ratio, $\sqrt{-1}=i$, and the last line holds for $x \ll 1$. Equation (15) diverges logarithmically for a light impurity with $\alpha<1$, when the Fermi energy of the impurities is resonant with the Bogoliubov spectrum, i.e., when $\epsilon_{F}=E_{k_{F}}$. This divergence reflects that the transferred momentum-energy between a polaron at the Fermi surface and the zero-momentum polaron is resonant with a sound mode in the BEC.

This divergence will be softened to a resonance in a real experiment for two reasons. First, any finite temperature smoothes out the Fermi surface. Second, the Bogoliubov modes are damped due to scattering on the impurities for a finite impurity concentration, which broadens the pole of the Landau interaction into a resonance as discussed above. However, for low temperatures and impurity concentrations, these effects are small and the energy shift remains large and nonmonotonic as a function of the impurity concentration.

In Fig. 9, we plot $\Delta \varepsilon(x)$ as a function of impurity concentration $x$ for unit mass ratio $m / m_{B}=1, k_{n} a_{B}=0.2$, and different boson-impurity scattering lengths. We take the temperature $T=0.4 T_{c}$ so that the bosonic impurities remain uncondensed for the range of concentrations shown. Figure 9 shows that the polaron energy decreases with an increasing impurity concentration. This decrease is caused by a mainly attractive Landau effective interaction between the bosonic polarons; see Fig. 3. We see that the concentration dependence of the energy increases with the

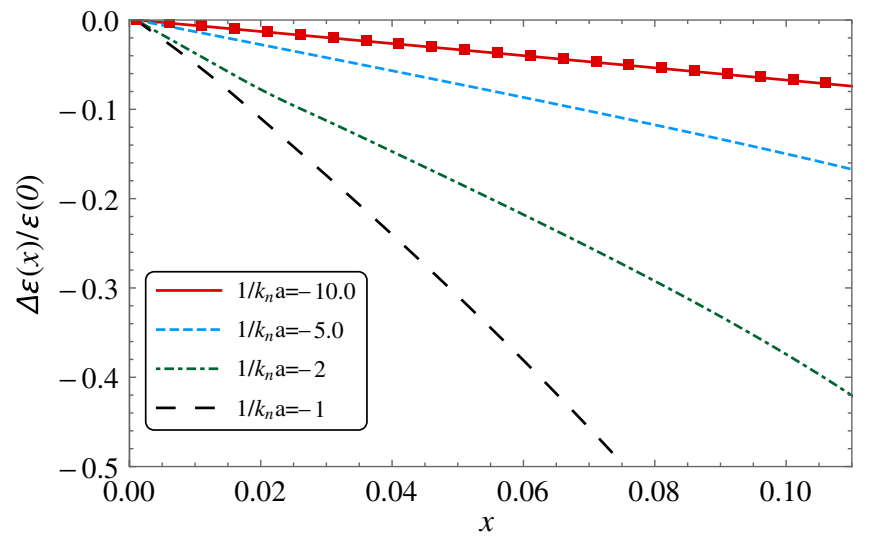

FIG. 9. The concentration dependence of the zero-momentum polaron energy Eq. (13) for $m / m_{B}=1, T=0.4 T_{c}, k_{n} a_{B}=0.2$, and various impurity-boson scattering lengths. We also show the perturbative result $\Delta \varepsilon(x)=-n \mathcal{T}_{v}^{2} / \mathcal{T}_{B}$ (red squares) for $1 / k_{n} a=-10$.

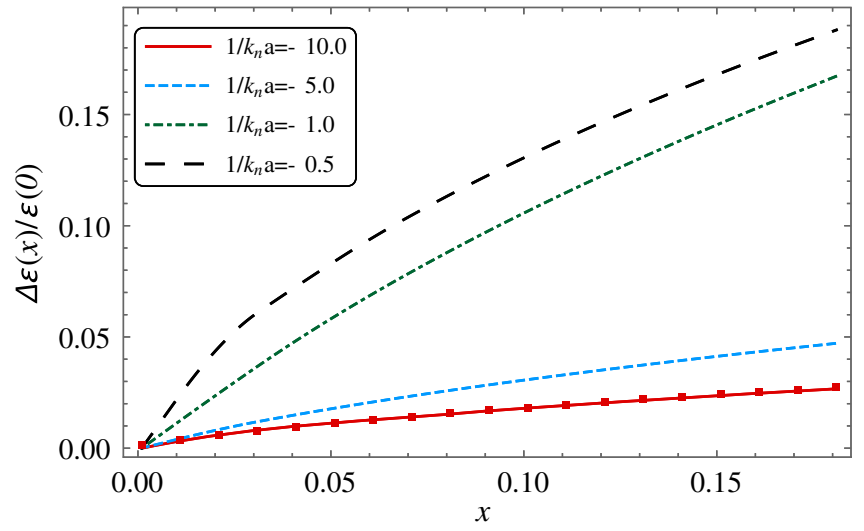

FIG. 10. The dependence $\Delta \varepsilon(x)$ of the zero-momentum polaron energy on the impurity concentration $x$ for mass ratio $\mathrm{m} / \mathrm{m}_{B}=$ $40 / 7, T=0, k_{n} a_{B}=0.2$, and various impurity-boson scattering lengths. We also show the perturbative result Eq. (15) for $1 / k_{n} a=-10$ (red squares).

boson-impurity scattering length $a$, even when it is measured in units of the polaron energy at zero impurity concentration $\varepsilon(0)-\mathrm{a}$ unit which, of course, also increases with $a$. In the weak-coupling limit, this increase is easily understood from the fact that the polaron mean-field energy $\mathcal{T}_{v} n_{B}$ scales linearly with $a$, whereas $\Delta \varepsilon(x)=$ $-n \mathcal{T}_{v}^{2} / \mathcal{T}_{B} \propto a^{2}$. This perturbative result is recovered for $1 / k_{n} a=-10$ as can be seen in Fig. 9. When the impurityboson interaction is strong, the decrease in the energy is significant. For $1 / k_{n} a=-1$, the decrease is around $50 \%$ compared to the polaron energy at a zero concentration already at $x \simeq 0.075$.

As an example of fermionic impurities, we plot in Fig. 10 the dependence of a zero-momentum polaron energy on the impurity concentration for the mass ratio $m / m_{B}=40 / 7$. In contrast to the case of bosonic impurities discussed above, the energy now increases with $x$, since the Landau effective interaction is predominantly repulsive between fermionic impurities (see Fig. 4). For a weak interaction with $1 / k_{n} a=-10$, the energy shift is well described by the perturbative result Eq. (15). For a stronger interaction, the concentration dependence of the energy of the fermionic impurities is significant. It is, however, suppressed compared to the case of bosonic impurities as can be seen by comparing Fig. 10 with Fig. 9. One reason is that Fermi blocking of the impurities decreases the effects of the Landau effective interaction.

\section{DISCUSSION}

Bose polarons have been observed both in the case of bosonic impurities [20] and for fermionic impurities [21]. These experiments focus on the detection of the single quasiparticle properties, namely, the energy and the damping of the polaron. Preliminary data concerning the concentration dependence of the polaron energy were, however, reported in the Aarhus experiment, which is 
precisely the experimental method for detecting the Landau effective interaction we analyze here. The experiments work with impurity concentrations up to around 10\%, which should give rise to observable shifts away from the single impurity limit as can be seen from Figs. 9 and 10. This shift should be contrasted with the case for Fermi polarons, where the Landau effective interaction has remained undetected so far in spite of concerted experimental efforts [11]. The reason for this difference is, of course, that the Landau interaction is much larger for Bose polarons than for Fermi polarons, as we discuss above. We note that, for an accurate measurement of the interaction, one must have good control of the temperature and impurity concentration. The effects of the trap inhomogeneity should, moreover, be reduced or even eliminated. We also note that current experimental breakthroughs with ultracold gases offer new exciting mixtures where both heavy and light impurities can be examined [48,52-55].

Since the effective interaction is mediated by sound modes in the BEC, the corresponding timescale for the effects of polaron-polaron interactions is set by the average distance between the polarons divided by the speed of sound in the BEC. In order to observe the Landau effective interaction between polarons, this timescale must be shorter than the lifetime of the polarons due to, for instance, threebody decay.

An important question concerns the accuracy of our theory in the challenging strongly interacting regime $k_{n}|a| \gtrsim 1$, where there is no small parameter. We have very recently benchmarked our theory against Monte Carlo calculations by calculating the binding energy of so-called bipolarons, which are dimer states of two polarons bound together by the induced interaction. It turns out that the binding energy calculated using the induced interaction obtained with the theory presented here agrees well with Monte Carlo results [56]. This agreement shows that our theory is, in fact, reliable, even for a strong impurity-boson interaction. We speculate that a reason for this accuracy, which is a priori not obvious, is that it systematically combines two theories which each have proven to be accurate. The boson-impurity scattering is described using the ladder approximation, which has turned out to be surprisingly accurate for cold atomic gases in the strongly interacting regime - in particular, for the polaron problem [9,11-19,21,27]. Moreover, the fact that the exchange of density oscillations is the main mechanism leading to an effective interaction in many-body systems has been applied successfully in a wide range of physical settings with strong interactions, including liquid-helium and condensed-matter systems. We describe the density oscillations in the weakly interacting BEC using Bogoliubov theory, which is known to be accurate.

Finally, we note that, in addition to the dependence of the polaron energy on the impurity concentration, the induced interaction has many other interesting effects.
As mentioned above, it can lead to the formation of dimer states of polarons-the so-called bipolarons [56,57]. The presence of bipolarons is a many-body effect, as they are bound by the induced interaction analyzed in this paper. Bipolarons therefore become unbound with a vanishing BEC density. They are distinct from Efimov states, which are an effect of three-body physics and therefore also stable in a vacuum $[24,40]$. On the other hand, bipolarons can be stable in the perturbative regime where no Efimov states occur. Another interesting effect is that, since the induced interaction is inherently attractive, it can give rise to pairing between fermionic impurities, leading to the formation of unconventional superfluid states [58-63].

\section{CONCLUSIONS}

We investigate the Landau effective interaction between Bose polarons for arbitrary coupling strengths and momenta. Using perturbation theory, we derive analytical results in the limit when the boson-impurity interaction is weak. We show that for light impurities the interaction can be strong even in the weak-coupling regime, when the transferred momentum and energy between the polarons is resonant with a sound mode in the BEC. To investigate the Landau interaction for arbitrary boson-impurity interaction strength, we develop a diagrammatic scheme that recovers the correct weak-coupling limit. We show that the interaction is large when the boson-impurity scattering is close to the unitarity limit or when the momentum of the polaron approaches the threshold for momentum relaxation in the BEC. The Landau interaction between Bose polarons is, in general, much stronger than between Fermi polarons due to the large compressibility of the BEC, and we show how this leads to a substantial shift in the polaron energy as a function of the impurity concentration. We conclude that this shift should be observable using present-day experimental technology.

Our results show how the great flexibility of cold atomic gases can be used to explore Landau's theory of quasiparticles systematically and in regimes never realized before. Extending the use of this theory is important, given that it forms a powerful platform for our description of many-body systems across a wide range of energy scales. Our theoretical scheme, which combines two theories each known to be accurate, turns out to be reliable even in the strongly interacting regime. It relies on the microscopic interaction being short range, which is indeed the case in many physical settings where screening effects are significant. Our scheme could therefore be useful for systems other than atomic gases.

\section{ACKNOWLEDGMENTS}

We acknowledge useful discussions with P. Naidon, L. Peña Ardila, C. J. Pethick, and T. Enss. We acknowledge the support of the Villum Foundation. 


\section{APPENDIX: SELF-ENERGY AND INDUCED INTERACTION}

The pair propagator $\Pi_{11}(p)$ reads as follows:

$$
\begin{aligned}
\Pi_{11}(p)= & \int \frac{d^{3} k}{(2 \pi)^{3}}\left[\frac{u_{\mathbf{k}}^{2}\left(1+n_{\mathbf{k}}^{B} \pm n_{\mathbf{k}+\mathbf{p}}\right)}{z-E_{\mathbf{k}}-\xi_{\mathbf{k}+\mathbf{p}}}\right. \\
& \left.+\frac{v_{\mathbf{k}}^{2}\left(n_{\mathbf{k}}^{B} \mp n_{\mathbf{k}+\mathbf{p}}\right)}{z+E_{\mathbf{k}}-\xi_{\mathbf{k}+\mathbf{p}}}+\frac{2 m_{r}}{k^{2}}\right],
\end{aligned}
$$

where $n_{\mathbf{k}}^{B}=1 /\left[\exp \left(E_{\mathbf{k}} / T\right)-1\right]$ denotes the bosonic distribution function of the reservoir for $T<T_{c}$ and the last term of $\Pi_{11}(p)$ regularizes the pair propagator [27].

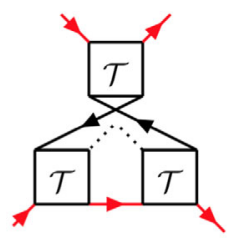

(a)

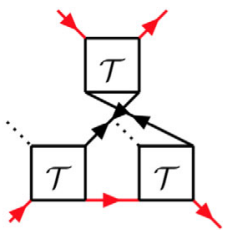

(b)

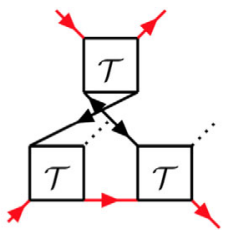

(c)

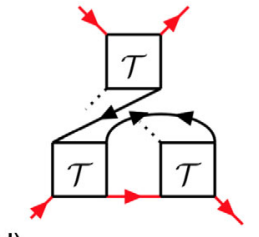

(d)

FIG. 11. Diagrams obtained from the dependence of the $\mathcal{T}$ matrices in Fig. 6 on the impurity density. We do not show the equivalent diagrams, where two $\mathcal{T}$ matrices are on top and one below.

Performing the Matsubara sums yields

$$
\begin{aligned}
\Sigma_{11}(p)= & -\frac{1}{\beta} \sum_{i \omega_{\nu}} \int \frac{d^{3} k}{(2 \pi)^{3}} G_{11}\left(\mathbf{k}, i \omega_{\nu}\right) n_{0} \mathcal{T}^{2}\left(\mathbf{k}+\mathbf{p}, i \omega_{\nu}+z\right) G\left(\mathbf{k}+\mathbf{p}, i \omega_{\nu}+z\right) \\
= & n_{0} \int \frac{d^{3} k}{(2 \pi)^{3}}\left[G\left(\mathbf{k}+\mathbf{p}, z+E_{\mathbf{k}}\right) u_{\mathbf{k}}^{2} n_{\mathbf{k}}^{B} \mathcal{T}^{2}\left(\mathbf{k}+\mathbf{p}, z+E_{\mathbf{k}}\right)+G\left(\mathbf{k}+\mathbf{p}, z-E_{\mathbf{k}}\right) v_{\mathbf{k}}^{2}\left(1+n_{\mathbf{k}}^{B}\right) \mathcal{T}^{2}\left(\mathbf{k}+\mathbf{p}, z-E_{\mathbf{k}}\right)\right] \\
& \pm n_{0} \int \frac{d^{3} k}{(2 \pi)^{3}} n_{\mathbf{k}+\mathbf{p}} \operatorname{Re}\left[\mathcal{T}^{2}\left(\mathbf{k}+\mathbf{p}, \xi_{\mathbf{k}+\mathbf{p}}\right)\right] G_{11}\left(\mathbf{k}, \xi_{\mathbf{k}+\mathbf{p}}-z\right) \\
& \mp n_{0} \int \frac{d^{3} k}{(2 \pi)^{3}} \int \frac{d \epsilon}{2 \pi} n_{\epsilon} \operatorname{Im}\left[2 \mathcal{T}^{2}(\mathbf{k}+\mathbf{p}, \epsilon)\right] G(\mathbf{k}+\mathbf{p}, \epsilon) G_{11}(\mathbf{k}, \epsilon-z)
\end{aligned}
$$

for the second term in (8) and

$$
\begin{aligned}
\Sigma_{12}(p)= & -\frac{2}{\beta} \sum_{i \omega_{\nu}} \int \frac{d^{3} k}{(2 \pi)^{3}} n_{0} \mathcal{T}(p) G_{12}(k) \mathcal{T}(k+p) G(k+p) \\
= & -2 n_{0} \mathcal{T}(p) \int \frac{d^{3} k}{(2 \pi)^{3}} u_{\mathbf{k}} v_{\mathbf{k}}\left[G\left(\mathbf{k}+\mathbf{p}, z+E_{\mathbf{k}}\right) n_{\mathbf{k}}^{B} \mathcal{T}\left(\mathbf{k}+\mathbf{p}, z+E_{\mathbf{k}}\right)+G\left(\mathbf{k}+\mathbf{p}, z-E_{\mathbf{k}}\right)\left(1+n_{\mathbf{k}}^{B}\right) \mathcal{T}\left(\mathbf{k}+\mathbf{p}, z-E_{\mathbf{k}}\right)\right] \\
& \pm 2 n_{0} \mathcal{T}(p) \int \frac{d^{3} k}{(2 \pi)^{3}} n_{\mathbf{k}+\mathbf{p}} \operatorname{Re}\left[\mathcal{T}\left(\mathbf{k}+\mathbf{p}, \xi_{\mathbf{k}+\mathbf{p}}\right)\right] G_{12}\left(\mathbf{k}, \xi_{\mathbf{k}+\mathbf{p}}-z\right) \\
& \mp 2 n_{0} \mathcal{T}(p) \int \frac{d^{3} k}{(2 \pi)^{3}} \int \frac{d \epsilon}{2 \pi} n_{\epsilon} \operatorname{Im}[2 \mathcal{T}(\mathbf{k}+\mathbf{p}, \epsilon)] G(\mathbf{k}+\mathbf{p}, \epsilon) G_{12}(\mathbf{k}, \epsilon-z)
\end{aligned}
$$

for the third term in (8). We define $n_{\mathbf{k}}=\left(\exp \beta \xi_{\mathbf{k}} \mp 1\right)^{-1}$ and $n_{\epsilon}=(\exp \beta \epsilon \mp 1)^{-1}$, where the upper (lower) sign as usual is for bosonic (fermionic) impurities.

The diagrams for the effective interaction coming from the dependence of the $\mathcal{T}$ matrices in Fig. 6 on the impurity concentration are shown in Fig. 11. They can be obtained by removing an impurity line inside one of the $\mathcal{T}$ matrices, which produces diagrams for the interaction with three $\mathcal{T}$ matrices. We do not include the diagrams in Fig. 11 when calculating the effective interaction, since they do not represent a consistent inclusion of all diagrams with three $\mathcal{T}$ matrices. 
[1] L. D. Landau, The Theory of a Fermi Liquid, J. Exp. Theor. Phys. 3, 920 (1957).

[2] L. D. Landau, Oscillations in a Fermi Liquid, J. Exp. Theor. Phys. 5, 101 (1957).

[3] G. Baym and C. Pethick, Landau Fermi-Liquid Theory: Concepts and Applications (Wiley-VCH, New York, 1991).

[4] J. R. Schrieffer, Theory of Superconductivity, Advanced Book Program Series (Avalon, New York, 1983).

[5] D. J. Scalapino, The Case for $d x 2$ y2 Pairing in the Cuprate Superconductors, Phys. Rep. 250, 329 (1995).

[6] S. Weinberg, The Quantum Theory of Fields, The Quantum Theory of Fields Vol. 1 (Cambridge University Press, Cambridge, England, 1995).

[7] C. Chin, R. Grimm, P. Julienne, and E. Tiesinga, Feshbach Resonances in Ultracold Gases, Rev. Mod. Phys. 82, 1225 (2010).

[8] A. Schirotzek, C.-H. Wu, A. Sommer, and M. W. Zwierlein, Observation of Fermi Polarons in a Tunable Fermi Liquid of Ultracold Atoms, Phys. Rev. Lett. 102, 230402 (2009).

[9] C. Kohstall, M. Zaccanti, M. Jag, A. Trenkwalder, P. Massignan, G. M. Bruun, F. Schreck, and R. Grimm, Metastability and Coherence of Repulsive Polarons in a Strongly Interacting Fermi Mixture, Nature (London) 485, 615 (2012).

[10] M. Koschorreck, D. Pertot, E. Vogt, B. Fröhlich, M. Feld, and M. Köhl, Attractive and Repulsive Fermi Polarons in Two Dimensions, Nature (London) 485, 619 (2012).

[11] F. Scazza, G. Valtolina, P. Massignan, A. Recati, A. Amico, A. Burchianti, C. Fort, M. Inguscio, M. Zaccanti, and G. Roati, Repulsive Fermi Polarons in a Resonant Mixture of Ultracold ${ }^{6} \mathrm{Li}$ Atoms, Phys. Rev. Lett. 118, 083602 (2017).

[12] F. Chevy, Universal Phase Diagram of a Strongly Interacting Fermi Gas with Unbalanced Spin Populations, Phys. Rev. A 74, 063628 (2006).

[13] N. Prokof'ev and B. Svistunov, Fermi-Polaron Problem: Diagrammatic Monte Carlo Method for Divergent SignAlternating Series, Phys. Rev. B 77, 020408 (2008).

[14] C. Mora and F. Chevy, Normal Phase of an Imbalanced Fermi Gas, Phys. Rev. Lett. 104, 230402 (2010).

[15] M. Punk, P. T. Dumitrescu, and W. Zwerger, Polaron-toMolecule Transition in a Strongly Imbalanced Fermi Gas, Phys. Rev. A 80, 053605 (2009).

[16] R. Combescot, S. Giraud, and X. Leyronas, Analytical Theory of the Dressed Bound State in Highly Polarized Fermi Gases, Europhys. Lett. 88, 60007 (2009).

[17] X. Cui and H. Zhai, Stability of a Fully Magnetized Ferromagnetic State in Repulsively Interacting Ultracold Fermi Gases, Phys. Rev. A 81, 041602(R) (2010).

[18] P. Massignan and G. M. Bruun, Repulsive Polarons and Itinerant Ferromagnetism in Strongly Polarized Fermi Gases, Eur. Phys. J. D 65, 83 (2011).

[19] P. Massignan, M. Zaccanti, and G. M. Bruun, Polarons, Dressed Molecules and Itinerant Ferromagnetism in Ultracold Fermi Gases, Rep. Prog. Phys. 77, 034401 (2014).

[20] N. B. Jørgensen, L. Wacker, K. T. Skalmstang, M. M. Parish, J. Levinsen, R. S. Christensen, G. M. Bruun, and J. J. Arlt, Observation of Attractive and Repulsive Polarons in a Bose-Einstein Condensate, Phys. Rev. Lett. 117, 055302 (2016).
[21] M.-G. Hu, M. J. Van de Graaff, D. Kedar, J. P. Corson, E. A. Cornell, and D.S. Jin, Bose Polarons in the Strongly Interacting Regime, Phys. Rev. Lett. 117, 055301 (2016).

[22] J. Catani, G. Lamporesi, D. Naik, M. Gring, M. Inguscio, F. Minardi, A. Kantian, and T. Giamarchi, Quantum Dynamics of Impurities in a One-Dimensional Bose Gas, Phys. Rev. A 85, 023623 (2012).

[23] W. Li and S. D. Sarma, Variational Study of Polarons in Bose-Einstein Condensates, Phys. Rev. A 90, 013618 (2014).

[24] J. Levinsen, M. M. Parish, and G. M. Bruun, Impurity in a Bose-Einstein Condensate and the Efimov Effect, Phys. Rev. Lett. 115, 125302 (2015).

[25] Y. E. Shchadilova, R. Schmidt, F. Grusdt, and E. Demler, Quantum Dynamics of Ultracold Bose Polarons, Phys. Rev. Lett. 117, 113002 (2016).

[26] J. Tempere, W. Casteels, M. K. Oberthaler, S. Knoop, E. Timmermans, and J. T. Devreese, Feynman Path-Integral Treatment of the BEC-Impurity Polaron, Phys. Rev. B 80, 184504 (2009).

[27] S. P. Rath and R. Schmidt, Field-Theoretical Study of the Bose Polaron, Phys. Rev. A 88, 053632 (2013).

[28] R. S. Christensen, J. Levinsen, and G. M. Bruun, Quasiparticle Properties of a Mobile Impurity in a Bose-Einstein Condensate, Phys. Rev. Lett. 115, 160401 (2015).

[29] F. Grusdt, R. Schmidt, Y. E. Shchadilova, and E. Demler, Strong-Coupling Bose Polarons in a Bose-Einstein Condensate, Phys. Rev. A 96, 013607 (2017).

[30] G. E. Astrakharchik and L. P. Pitaevskii, Motion of a Heavy Impurity through a Bose-Einstein Condensate, Phys. Rev. A 70, 013608 (2004).

[31] F. M. Cucchietti and E. Timmermans, Strong-Coupling Polarons in Dilute Gas Bose-Einstein Condensates, Phys. Rev. Lett. 96, 210401 (2006).

[32] A. G. Volosniev, H.-W. Hammer, and N. T. Zinner, RealTime Dynamics of an Impurity in an Ideal Bose Gas in a Trap, Phys. Rev. A 92, 023623 (2015).

[33] R. Schmidt, H. R. Sadeghpour, and E. Demler, Mesoscopic Rydberg Impurity in an Atomic Quantum Gas, Phys. Rev. Lett. 116, 105302 (2016).

[34] L. A. Peña Ardila and S. Giorgini, Impurity in a BoseEinstein Condensate: Study of the Attractive and Repulsive Branch Using Quantum Monte Carlo Methods, Phys. Rev. A 92, 033612 (2015).

[35] L. A. Peña Ardila and S. Giorgini, Bose Polaron Problem: Effect of Mass Imbalance on Binding Energy, Phys. Rev. A 94, 063640 (2016).

[36] Z. Yu and C. J. Pethick, Induced Interactions in Dilute Atomic Gases and Liquid Helium Mixtures, Phys. Rev. A 85, 063616 (2012).

[37] H. Hu, B. C. Mulkerin, J. Wang, and X.-J. Liu, Attractive Fermi Polarons at Nonzero Temperature with Finite Impurity Concentration, Phys. Rev. A 98, 013626 (2018).

[38] A. S. Dehkharghani, A. G. Volosniev, and N. T. Zinner, Interaction-Driven Coalescence of Two Impurities in a One-Dimensional Bose Gas, arXiv:1712.01538 [Phys. Rev. Lett. (to be published)].

[39] A.Klein and M. Fleischhauer, Interaction of Impurity Atoms in Bose-Einstein Condensates, Phys. Rev. A 71, 033605 (2005). 
[40] P. Naidon, Two Impurities in a Bose-Einstein Condensate: From Yukawa to Efimov Attracted Polarons, J. Phys. Soc. Jpn. 87, 043002 (2018).

[41] J. Levinsen, M. M. Parish, R. S. Christensen, J. J. Arlt, and G. M. Bruun, Finite-Temperature Behavior of the Bose Polaron, Phys. Rev. A 96, 063622 (2017).

[42] Z. Yu, S. Zöllner, and C. J. Pethick, Comment on Normal Phase of an Imbalanced Fermi Gas, Phys. Rev. Lett. 105, 188901 (2010).

[43] Technically, this term comes from the pole of the induced interaction, which does not occur in an exchange diagram with an ordinary frequency-independent interaction.

[44] L. Viverit, C. J. Pethick, and H. Smith, Zero-Temperature Phase Diagram of Binary Boson-Fermion Mixtures, Phys. Rev. A 61, 053605 (2000).

[45] H. Shi and A. Griffin, Finite-Temperature Excitations in a Dilute Bose-Condensed Gas, Phys. Rep. 304, 1 (1998).

[46] S. Watabe and Y. Ohashi, Comparative Studies of ManyBody Corrections to an Interacting Bose-Einstein Condensate, Phys. Rev. A 88, 053633 (2013).

[47] A. R. Fritsch, P. E. S. Tavares, F. A. J. Vivanco, G. D. Telles, V. S. Bagnato, and E. A. L. Henn, Thermodynamic Measurement of the Sound Velocity of a Bose Gas across the Transition to Bose-Einstein Condensation, J. Stat. Mech. (2018), 053108.

[48] R. S. Lous, I. Fritsche, M. Jag, B. Huang, and R. Grimm, Thermometry of a Deeply Degenerate Fermi Gas with a Bose-Einstein Condensate, Phys. Rev. A 95, 053627 (2017).

[49] A. Guidini, G. Bertaina, D. E. Galli, and P. Pieri, Condensed Phase of Bose-Fermi Mixtures with a Pairing Interaction, Phys. Rev. A 91, 023603 (2015).

[50] N.-E. Guenther, P. Massignan, M. Lewenstein, and G. M. Bruun, Bose Polarons at Finite Temperature and Strong Coupling, Phys. Rev. Lett. 120, 050405 (2018).

[51] D. Suchet, Z. Wu, F. Chevy, and G. M. Bruun, Long-Range Mediated Interactions in a Mixed-Dimensional System, Phys. Rev. A 95, 043643 (2017).

[52] Y.-P. Wu, X.-C. Yao, H.-Z. Chen, X.-P. Liu, X.-Q. Wang, Y.-A. Chen, and J.-W. Pan, A Quantum Degenerate
Bose-Fermi Mixture of $41 \mathrm{~K}$ and $6 \mathrm{Li}$, J. Phys. B 50, 094001 (2017).

[53] J. W. Park, S. A. Will, and M. W. Zwierlein, Ultracold Dipolar Gas of Fermionic ${ }^{23} \mathrm{Na}^{40} \mathrm{~K}$ Molecules in Their Absolute Ground State, Phys. Rev. Lett. 114, 205302 (2015).

[54] T. A. Schulze, T. Hartmann, K. K. Voges, M. W. Gempel, E. Tiemann, A. Zenesini, and S. Ospelkaus, Feshbach Spectroscopy and Dual-Species Bose-Einstein Condensation of ${ }^{23} \mathrm{Na}-{ }^{39} \mathrm{~K}$ Mixtures, Phys. Rev. A 97, 023623 (2018).

[55] M.-S. Heo, T. T. Wang, C. A. Christensen, T. M. Rvachov, D. A. Cotta, J.-H. Choi, Y.-R. Lee, and W. Ketterle, Formation of Ultracold Fermionic Nali Feshbach Molecules, Phys. Rev. A 86, 021602 (2012).

[56] A. Camacho-Guardian, L. A. Peña Ardila, T. Pohl, and G. M. Bruun, Bipolarons in a Bose-Einstein Condensate, Phys. Rev. Lett. 121, 013401 (2018).

[57] J. T. Devreese and A. S. Alexandrov, Fröhlich Polaron and Bipolaron: Recent Developments, Rep. Prog. Phys. 72, 066501 (2009).

[58] H. Heiselberg, C. J. Pethick, H. Smith, and L. Viverit, Influence of Induced Interactions on the Superfluid Transition in Dilute Fermi Gases, Phys. Rev. Lett. 85, 2418 (2000).

[59] D. V. Efremov and L. Viverit, $p$-Wave Cooper Pairing of Fermions in Mixtures of Dilute Fermi and Bose Gases, Phys. Rev. B 65, 134519 (2002).

[60] F. Illuminati and A. Albus, High-Temperature Atomic Superfluidity in Lattice Bose-Fermi Mixtures, Phys. Rev. Lett. 93, 090406 (2004).

[61] K. Suzuki, T. Miyakawa, and T. Suzuki, $p$-Wave Superfluid and Phase Separation in Atomic Bose-Fermi Mixtures, Phys. Rev. A 77, 043629 (2008).

[62] T. Enss and W. Zwerger, Superfluidity near Phase Separation in Bose-Fermi Mixtures, Eur. Phys. J. B 68, 383 (2009).

[63] Z. Wu and G. M. Bruun, Topological Superfluid in a FermiBose Mixture with a High Critical Temperature, Phys. Rev. Lett. 117, 245302 (2016). 